\title{
Advanced nanomaterials targeting hypoxia to enhance radiotherapy
}

This article was published in the following Dove Press journal:

International Journal of Nanomedicine

\author{
Jia $\mathrm{Li}^{\mathrm{I}-3, *}$ \\ Wenting Shang $2,3, *$ \\ Yong $\mathrm{Li}^{1}$,* \\ Sirui $\mathrm{Fu}^{\mathrm{I}-3}$ \\ Jie Tian 2,3 \\ Ligong Lu'
}

'Zhuhai Precision Medical Center, Zhuhai People's Hospital, Jinan University, Zhuhai, China; ${ }^{2}$ CAS Key Laboratory of Molecular Imaging, Institute of Automation, Chinese Academy of Sciences, Beijing, China; ${ }^{3}$ Beijing Key Laboratory of Molecular Imaging, Beijing, China

*These authors contributed equally to this work
Correspondence: Ligong Lu

Zhuhai People's Hospital, 95 Kangning

Road, Xiangzhou District, Guangdong

Province, Zhuhai 519000, China

Tel +8607562I582II

Email luligong1969@126.com

Jie Tian

Beijing Key Laboratory of Molecular Imaging, 95 Zhongguancun East Road, Haidian District, Beijing 100190, China

Tel +861082618465

Fax +861062527995

Email jie.tian@ia.ac.cn

\begin{abstract}
Hypoxia within solid tumors is often responsible for the failure of radiotherapy. The development of hypoxia-targeting nanomaterials - aimed at enhancing the effect of radiotherapy by electrical or heat effects and at modulating hypoxia in the tumor microenvironment - is a promising strategy to address this issue. We provide an overview of recently developed advanced materials that potentiate radiotherapy. First, we summarize novel materials for oxygen delivery or production to modify the tumor microenvironment, thus improving the effects of ionizing radiation. Second, we present new approaches for the design of high- $Z$ element-based multifunctional nanoplatforms to enhance radiotherapy. Third, novel drug delivery systems for hypoxic regions and hypoxia-inducible factor-1-targeted therapies are discussed. Fourth, we establish the effectiveness of X-ray- or near-infrared-responsive nanoparticles for selectively triggering therapeutic effects under hypoxic conditions. Finally, this review emphasizes the importance of research in the field of nanomedicine focused on tumor hypoxia to improve clinical outcomes.
\end{abstract}

Keywords: radiotherapy, hypoxia, nanomaterials, hypoxia-inducible factor 1

\section{Introduction}

Tumor hypoxia is defined as a low $\mathrm{O}_{2}$ concentration in a specific tumor region. Because most tumor cells proliferate exponentially, aberrant vasculature inside the tumor generates an imbalance between the $\mathrm{O}_{2}$ supply and consumption, together with an increase in diffusion distances, eventually making the central tumor area hypoxic. Vaupel et $\mathrm{al}^{1,2}$ first detected hypoxia in breast tumors using an electrode probe. Emerging evidence has demonstrated that tumor cells under hypoxia are typically resistant to anticancer drugs and external beam radiation. ${ }^{3-6}$ Moreover, hypoxia promotes genetic transformation into a more invasive cell phenotype, thus potentiating metastasis and recurrence. . $^{3,4}$

Various phenomena are closely associated with the physiological and anatomical characteristics of hypoxic tumors. 1) The intratumoral hypoxic region is often distant from blood capillaries $(>100 \mu \mathrm{m})$, which is challenging for the penetration and distribution of the majority of anticancer drugs. ${ }^{5}$ 2) The overexpression of hypoxia-inducible factor-1 (HIF-1) activates the transcription of multiple genes involved in the epithelialmesenchymal transition, angiogenesis, and proliferation of tumors, ${ }^{4,6,7}$ suggesting the importance of HIF-1-targeted therapies (see HIF-1-targeted therapy). 3) Insufficient oxygen hampers the formation of oxidation radicals, resulting in permanent tumor cell damage; in contrast, excessive reduction groups, such as $\mathrm{SH}-$, may facilitate the repair of tumor cell DNA. ${ }^{8} 4$ ) Hypoxic tumor cells depend on aerobic glycolysis (the Warburg effect); thus, the tumor microenvironment (TME) is characterized by 
a low $\mathrm{pH}$, which may have synergistic effects with hypoxia on malignant transformation. ${ }^{8,9}$ Overall, tumor hypoxia is a major obstacle to the long-standing goal of developing effective therapies for cancer.

Broadly speaking, radiotherapy has become a common therapeutic strategy, applied to $>50 \%$ of patients with cancer. ${ }^{10}$ Radiotherapy is based on the use of high-energy $\mathrm{X}$-rays and gamma $(\gamma)$ rays for external or internal irradiation by means of radiolabeled isotopes, such as ${ }^{131} \mathrm{I}$, carbon ions, or $\alpha$ - or $\beta$-particles. Radiation induces doublestranded DNA breaks and creates massive quantities of free radicals, which are lethal to most tumor cells. ${ }^{8}$ However, free radicals have minimal effects on solid tumors owing to hypoxia, which has a negative effect on the mechanism underlying damage by free radicals. On one hand, hypoxia attenuates oxidation to protect tumor cells from extensive damage; on the other hand, reductant chemical groups, such as $-\mathrm{SH}$, contribute to the repair of DNA, and this mechanism helps tumor cells survive postirradiation. ${ }^{8,11}$ Consequently, tumors under hypoxia are insensitive to radiotherapy.

Although hypoxia diminishes the effects of radiotherapy to some extent, novel radiosensitizers and intelligent nanomaterials offer synergistic approaches to fight against tumors. In this review, we summarize advances in biomedical engineering resulting in improvements in radiotherapy (Scheme 1). First, we discuss innovative approaches to the sensitization of tumors via the production or transportation of $\mathrm{O}_{2}$ in the TME. Second, we discuss high- $Z$ element-based nanoplatforms and synergistic drug delivery systems for radiosensitization. Third, we focus on recent advances in synergistic photothermal therapy and radiotherapy as well as $\mathrm{X}$-ray-responsive nanoparticles (NPs) for selective hypoxiabased therapy. Last, we summarize recent advances and discuss future perspectives regarding the use of nanomaterials for radiosensitization.

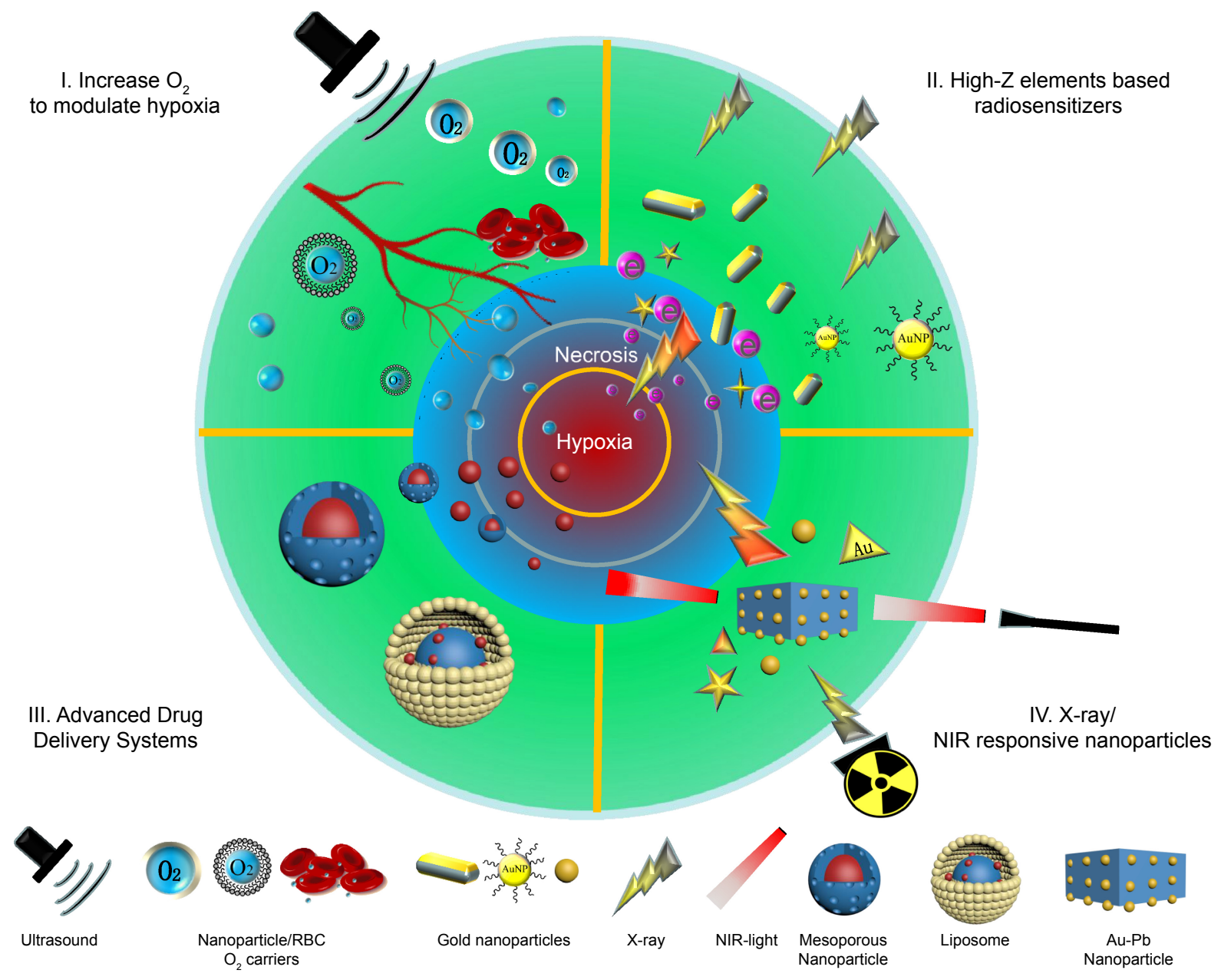

Scheme I Strategies for modifying tumor hypoxia.

Notes: I. Increase $\mathrm{O}_{2}$ directly to modulate hypoxia; II. High-Z element-based radiosensitizers; III. Advanced drug delivery systems; IV. X-ray/NIR-responsive nanoparticles. Abbreviation: NIR, near-infrared. 


\section{Increasing radiosensitivity by modulating hypoxia}

The TME is characterized by special features, such as a low $\mathrm{pH}$, inadequate $\mathrm{O}_{2}$ supply, and aberrant vasculature, among which hypoxia is directly related to radioresistance. ${ }^{6}$ To address this issue, recent efforts have been made to use classic oxygen carriers or to develop novel materials to reoxygenate the TME, thus increasing the radiosensitivity of tumors.

\section{Perfluorocarbons (PFCs) as $\mathrm{O}_{2}$ carriers}

As representative oxygen carriers, $\mathrm{PFCs}$ and their derivatives (perfluorotributylamines) are widely used as $\mathrm{O}_{2}$ carriers and tumor radiosensitizers owing to their rapid hydrolysis and high solubility in cellular microenvironments. ${ }^{12,13}$ As early as 1989, PFC emulsions (such as Fluosol-DA, 20\%) were approved by the Food and Drug Administration for ophthalmological treatments and percutaneous transluminal coronary angiography. ${ }^{14}$ To assess the efficacy of PFCs in reoxygenating the TME, Teicher et a ${ }^{15}$ administered a perflubron emulsion $(8 \mathrm{~mL} / \mathrm{kg})$ with carbogen breathing $\left(95 \% \mathrm{O}_{2} / 5 \%\right.$ $\mathrm{CO}_{2}$ ) to tumor-bearing mice immediately after chemo-/radiotherapy; remarkably, the hypoxic region $\left(\mathrm{PO}_{2} \leq 5 \mathrm{mmHg}\right)$ decreased from $85 \%$ to $27 \%$ due to reoxygenation. More recently, Feldman et a ${ }^{16}$ obtained similar results for GL261 intracranial tumors. Using another strategy, Yao et al ${ }^{7}$ developed a poly(lactic-co-glycolic acid) (PLGA)-(polyethylene glycol) (PEG/PFC) emulsion to achieve highly effective and rapid reoxygenation in cells and organisms; moreover, they indicated the potential for hypoxia-reoxygenation injury, which may induce the massive production of reactive oxygen species (ROS), harming normal tissues. This has limited the PEG/PFC emulsion dosage to below $0.2 \mathrm{mg} / \mathrm{mL}$. Furthermore, intermediate oxygen levels $(0.5-20 \mathrm{mmHg})$ regulate tumor responses to radiotherapy ${ }^{17}$ In addition to the loading capacity, the output stability of $\mathrm{O}_{2}$ should be considered to improve radiosensitization; in particular, a moderate increase in $\mathrm{O}_{2}$ levels is preferable for clinical outcomes. Additionally, abnormal vascular structure may make $\mathrm{O}_{2}$ diffusion from the blood to the hypoxic area difficult, and temporary blocks in the tumor vasculature limit effective accumulation, ${ }^{8}$ necessitating more effective surface modifications to target the tumor.

Multiple remarkable $\mathrm{O}_{2}$ nanoplatforms for sustained release have been developed in the past decade. Lee et $\mathrm{al}^{18}$ loaded a perfluorooctane emulsion into hollow microparticles to form a scaffolding system for timely $\mathrm{O}_{2}$ supply. An in vitro experiment revealed a 10-day extension of cell viability under hypoxic conditions and neovascularization of tissues up to $4 \mathrm{~mm}$, instead of necrosis. Cheng et a ${ }^{19}$ demonstrated more effective particle accumulation and specific $\mathrm{O}_{2}$ release in tumors to overcome hypoxia-related radioresistance. In brief, they encapsulated perfluorohexane within liposomes to form $100 \mathrm{~nm}$ NPs by a facile method and then injected these NPs into tumor-bearing mice prior to photodynamic therapy or radiotherapy. Considerable tumor inhibition was observed in both treatment groups compared with the control group. It is worth noting that this result was obtained without the supply of additional oxygen because the oxygen loaded inside the NPs was sufficient to neutralize hypoxia, mainly due to the enhanced permeability and retention (EPR) effect caused by an appropriate NP size and free oxygen diffusion inside the NPs. The development of promising $\mathrm{O}_{2}$ delivery materials is clearly favorable for therapeutic approaches, such as radiotherapy or photodynamic therapy. The combination of reoxygenation with radiosensitizers may be effective in vivo. Song et $\mathrm{a}^{20}$ developed a type of hollow $\mathrm{Bi}_{2} \mathrm{Se}_{3}$ NPs loaded with $\mathrm{PFC}$ as an $\mathrm{O}_{2}$ carrier. In addition to the radiosensitizing effects of bismuth, this system possesses high absorbance in the near-infrared (NIR) region, which could be utilized for photothermal therapy and the selective triggering of rapid $\mathrm{O}_{2}$ release. A synergistic therapeutic effect was detected in vivo. They next decorated tantalum oxide $\left(\mathrm{TaO}_{\mathrm{x}}\right) \mathrm{NPs}$ with PFCs for further enhancement of radiotherapy. ${ }^{21}$ Nevertheless, considering the limited penetration depth of NIR light and absorption by the skin and major organs, a more flexible and sensitive mechanism may be required to trigger the action of these composite NPs at safe laser intensities to avoid hyperthermia in normal tissues. Overall, this is a promising method for future clinical applications.

\section{Ultrasound-based $\mathrm{O}_{2}$ carriers}

Ultrasound combined with $\mathrm{O}_{2}$ carriers, such as microbubbles or NPs, has been utilized to distribute $\mathrm{O}_{2}$ to hypoxic regions. For example, microbubbles comprising multiple hollow structures can serve as $\mathrm{O}_{2}$ carriers and as ultrasonography contrast agents; ${ }^{22}$ additionally, multiple surface modifiers, such as phospholipids, ${ }^{23}$ chitosan, ${ }^{24}$ and proteins, ${ }^{25}$ have been used to prolong the $\mathrm{O}_{2}$ supply and enhance foam stability. Eisenbrey et $\mathrm{a}^{25}$ generated a novel microbubble $\left(\mathrm{SE} 61 \mathrm{O}_{2}\right)$ by mixing Span 60 with water-soluble vitamin E purged with PFC gas, followed by PFC removal and reconstitution with $\mathrm{O}_{2}$. Ultrasonography was used to visualize microbubbles and to trigger $\mathrm{O}_{2}$ release in hypoxic areas. In vivo results showed increases in oxygen levels of 30.4 and $27.4 \mathrm{mmHg}$ in two breast tumor mouse xenografts. Because microbubbles can be imaged by ultrasonography, Kwan et $\mathrm{al}^{26}$ delivered 
$\mathrm{O}_{2}$ to hypoxic regions via microbubbles, visualized real-time oxygen transport, and destroyed the microbubbles to release $\mathrm{O}_{2}$ by controlling the ultrasound intensity. Recently, Song et $\mathrm{al}^{27}$ developed a novel nanodroplet $\mathrm{O}_{2}$ shuttle that facilitates tumor-specific $\mathrm{O}_{2}$ delivery on the basis of low-intensity ultrasound. In brief, nanodroplets are loaded with $\mathrm{O}_{2}$ in the lungs and release $\mathrm{O}_{2}$ in tumors with the help of ultrasound, and then they flow back to lungs to reoxygenate in a cyclical manner. It is worth noting that the NPs were assembled from agents approved for clinical use, thus avoiding the issue of toxicity, which limited the materials that can be used for subsequent clinical application. Because ultrasound-based methods are noninvasive and nonradioactive techniques for tumor detection, ultrasound-based nanosystems are practical for clinical integration with radiotherapy. This innovative strategy has great potential for further clinical applications.

\section{Red blood cell (RBC)-based $\mathrm{O}_{2}$ carriers}

Another vital issue is that most nanomaterials encounter numerous biological barriers, such as the blood-brain barrier and nonspecific uptake by the reticuloendothelial system (RES) and mononuclear phagocyte system. ${ }^{28}$ Although multiple compounds, such as PEG and PLGA, have been utilized to prevent NP uptake by the RES, immune escape is difficult when multiple administration is required. ${ }^{29} \mathrm{RBCs}$, also known as erythrocytes, have been used as practical endogenous carriers for drug delivery since 1970 owing to their excellent biocompatibility, long circulation times ( $>120$ days), and unique loading capacity (high surface-tovolume ratio). To utilize these features for $\mathrm{O}_{2}$ delivery and improve the efficiency of radiotherapy, Gao et a ${ }^{30}$ developed PFC@PLGA-RBCM NPs. The highlight of this study was that this nanosized RBC mimic was able to penetrate the vasculature, diffusing deep within the hypoxic areas in solid tumors; furthermore, the NP half-life was extended to 13.93 hours, which significantly increased oxygenation levels from $1.6 \%$ to $24 \%$. Similarly, Ren et al ${ }^{31}$ encapsulated NIR dyes and a PFC into erythrocyte-attached NPs and observed a half-life of 15.71 hours, enabling the inhibition of tumor growth by photodynamic therapy. Although RBC-NP conjugates hold great promise for future applications, there are two major obstacles: 1) the loaded cargo may reduce $\mathrm{RBC}$ membrane elasticity, resulting in a risk of damage due to osmotic stress and a serious risk of blocking small vessels in kidneys or lungs due to released hemoglobin; 2) RBCs collected from individuals with an incompatible blood type may induce acute hemolysis. It is preferable to generate RBC-NPs from a patient's own blood sample.

\section{$\mathrm{H}_{2} \mathrm{O}_{2}$ catalyst-based nanoplatforms for reoxygenation}

There is strong evidence that endogenous $\mathrm{H}_{2} \mathrm{O}_{2}$ is present in major solid tumors. Additionally, excessive lactic acid levels are produced by tumor cells owing to the "Warburg effect," generating another low $\mathrm{pH}$ feature of the TME. ${ }^{32}$ Fortunately, $\mathrm{MnO}_{2}$ possesses excellent catalytic properties to decompose $\mathrm{H}_{2} \mathrm{O}_{2}$ into $\mathrm{O}_{2}$ and $\mathrm{H}_{2} \mathrm{O}$ under acidic conditions; therefore, it has been utilized as a vital component of various nanoplatforms intended to reoxygenate hypoxic tumors and improve therapeutic efficacy. ${ }^{33-36}$ As a remarkable example, Fan et al achieved simultaneous stimuli-responsive imaging and $\mathrm{O}_{2}$-upregulating therapy on a single nanoplatform. In brief, they successfully anchored upconversion NPs (UCNPs) to two-dimensional $\mathrm{MnO}_{2}$ nanosheets by a facile nonchemical method. While upconversion photoluminescence was quenched by $\mathrm{MnO}_{2}$ under normal conditions, a remarkable enhancement of upconversion photoluminescence was observed in the tumor region, and this can be explained by the acceleration of the $\mathrm{MnO}_{2}-\mathrm{H}_{2} \mathrm{O}_{2}$ redox reaction at a low $\mathrm{pH}$ (5.5 vs 7.4). More importantly, massive oxygen produced during this process significantly enhanced the simultaneous photodynamic therapy and radiotherapy, and this effect was confirmed in subsequent in vivo and in vitro studies. ${ }^{37}$

Additionally, because high- $Z$ elements possess the intrinsic ability to enhance ionizing radiation, recent studies have focused on combining a catalyst and a high- $Z$ element, thereby generating a series of intelligent nanoplatforms incorporating $\mathrm{H}_{2} \mathrm{O}_{2}$ catalysts with high- $Z$ elements, such as tungsten $\left(\mathrm{WS}_{2}-\mathrm{IO} / \mathrm{S} @ \mathrm{MO}-\mathrm{PEG}\right),{ }^{38}$ gold $\left(\mathrm{Au} @ \mathrm{MnO}_{2}\right),{ }^{39}$ or hafnium (BM@NCP(DSP)-PEG), ${ }^{40}$ for multimodal photodynamic therapy/radiation therapy/chemotherapy. For example, Tian et al ${ }^{36}$ developed ${ }^{131} \mathrm{I}$-labeled human serum albumin (HSA)-bound $\mathrm{MnO}_{2}$ NPs for internal radiotherapy. These NPs showed higher EPR-derived tumor retention than that of free ${ }^{131} \mathrm{I}$-HSA by optimizing the particle size. Moreover, by gradual $\mathrm{MnO}_{2}$ degradation in the acidic TME, the NP size could be sequentially reduced to $10 \mathrm{~nm}$. The intratumoral diffusion of the HSA-based nanocarrier improved significantly; additionally, endogenous $\mathrm{H}_{2} \mathrm{O}_{2}$ was converted into $\mathrm{H}_{2} \mathrm{O}$ and $\mathrm{O}_{2}$, and the latter remarkably increased the efficacy of radiotherapy. As mentioned above, endogenous $\mathrm{H}_{2} \mathrm{O}_{2}$ within the TME may be used to improve 
tumor oxygenation and has great potential for optimizing the efficacy of radiotherapy.

\section{High-Z element-based radiosensitizers and intelligent nanoplatforms for drug delivery Basic mechanism underlying high- $Z$ element-based radiosensitization}

Radiotherapy kills tumor cells by the action of ionizing radicals, commonly generated during external beam radiation therapy (EBRT) or internal beam radioisotope therapy (IBRT). In EBRT, high-energy X-rays are focused externally on the tumor to induce cell death, taking into account side effects on normal tissues. EBRT requires a precise angle of irradiation and quantification of the exposed region. EBRT may be used for the treatment of most tumors, such as brain, head and neck, lung, and esophageal cancers. In IBRT, a certain number of radioisotopic particles can be implanted inside the tumor by noninvasive or minimally invasive methods, thus exerting ionization effects within the tumor, which persist for a long time and may be suitable for certain cancers, such as thyroid cancer, hepatic cancer with portal vein thrombosis, or metastatic lesions.

Nonetheless, two major problems limit the actual effects of radiotherapy. First, the hypoxic environment prevents ROS formation, thus limiting the effects of ionization. Although a certain number of tumor cells may be eliminated, resident lesions still possess the ability to relapse or metastasize. ${ }^{8}$ Second, cancerous and normal cells surrounding the tumor cannot be precisely discriminated by ionizing radiation, necessitating dose limits. ${ }^{10}$

To overcome these issues, numerous radiosensitizers have been developed. As the pioneer G.E. Adams suggested in $1978,{ }^{41}$ radiosensitizers must greatly increase the efficacy of radiation-induced cell killing when present during the radiation; however, they should cause limited or no cell killing in the absence of radiation, implying that the response of tumors to radiation must be much greater than that of normal tissues. High- $Z$ elements, mainly gold $(Z=79)$, bismuth $(Z=83)$, tungsten $(Z=74)$, and rare earth elements, such as gadolinium $(Z=64)$ and ytterbium $(Z=70)$, have been widely used as radiosensitizers for many years owing to the good absorption of ionizing radiation within solid tumors. Two main mechanisms are responsible for their radiosensitizing effects: 1) a photoelectric effect, which is usually accompanied by Auger electrics and 2) the Compton effect. Photoelectric effects occur in major high- $Z$ elements under photon irradiation, whereby electrons are ejected from higher orbits to refill vacant spots and release energy in the form of free electrons or Auger electrons. The strength of the effect is proportional to the $Z$ number; therefore, high- $Z$ elements, such as gold $(Z=79)$ and bismuth $(Z=83)$, or the rare earth elements, such as gadolinium $(Z=64)$ and ytterbium $(Z=70)$, have been used. Meanwhile, Compton scattering is an inelastic collision, whereby only partial energy from incoming X-rays is distributed to electrons. Therefore, scattering $\mathrm{X}$-rays with low energy could remain within tumor tissues. Under general conditions, radiosensitization enhances the abovementioned mechanisms by elevating ROS production, increasing oxidative stress, and promoting DNA damage by chemical interactions.

\section{Modifying the surface, size, or shape of gold-based NPs (AuNPs) for enhanced radiosensitization}

In the past decade, AuNPs were popular radiosensitizers in clinical oncology. Cui et $\mathrm{al}^{42}$ demonstrated that the effect of radiosensitization by AuNPs is closely related to cellular uptake. Early studies revealed that cellular AuNP uptake proceeds via endocytic pathways, such as macropinocytosis ${ }^{43,44}$ and passive membrane diffusion, ${ }^{45}$ highlighting the necessity of surface coating to finetune endocytic AuNP uptake. ${ }^{46}$ Of note, Jain et $\mathrm{a}^{47}$ observed that receptor-mediated endocytosis is energy-dependent, and AuNP uptake under hypoxia may be related to anaerobic glycolysis, which produces much less energy. To reduce nonspecific AuNP uptake by normal tissues and organs, in which protein opsonization is commonly believed to play a major role, multiple passive-transporting agents - usually PEG - are used to protect the nanomaterials from phagocytic cells. Nevertheless, the results of van Haute et $\mathrm{al}^{48}$ contradicted previous views; they found that most nonspecific NP uptake can be attributed to scavenger receptors and NPs rather than to protein opsonization. These data may contribute to the development of novel surface modifications to effectively reduce nonspecific uptake and to enhance the capacity for targeting to hypoxic areas.

Additionally, many studies have shown that the structure and size of AuNPs, such as nanorods, ${ }^{49}$ nanospikes, ${ }^{50}$ nanoclusters, ${ }^{51}$ and nanotriangles, ${ }^{52}$ are correlated with their biological distribution, radiosensitizing effect, and cytotoxicity. Dou et $\mathrm{al}^{53}$ observed that AuNPs approximately $13 \mathrm{~nm}$ in size possess optimal radiosensitizing and superior computed tomography (CT) contrast ability compared with others. Ma et $\mathrm{al}^{54}$ compared performance between gold NPs, 
gold nanospikes, and gold nanorods. They observed that gold NPs possess higher anticancer efficiency in terms of radiosensitization (SER $=1.62)$ than those with the other shapes (SER $=1.37$ for gold nanospikes and 1.21 for gold nanorods). Subsequent results have attributed this phenomenon to effective cellular uptake. The shape of AuNPs can influence their radiosensitizing ability via cell uptake as well as ROS generation and cell-cycle redistribution, and gold NPs exert cytotoxic effects via apoptosis. In a more recent study, ${ }^{50}$ greater uptake of AuNPs was observed after coating with the tumor-cell-penetrating peptide TAT, with an SER of 2.30. Additionally, autophagy may perform an important function in cell resistance to ionizing radiation, as evidenced by the improvement in the effect of radiotherapy by autophagy inhibitors. The relation between autophagy and hypoxia radioresistance is not well established, and this should be a focus of future research in this field.

\section{Synergistic chemotherapy and radiotherapy for improving therapeutic outcomes}

The utilization of chemotherapy with radiotherapy to improve therapeutic efficacy and to prevent recurrence or metastasis has a long history. Multimodal clinical trials have revealed superior efficacy in comparison with single treatment modalities. ${ }^{55-57}$ Emerging nanomaterials possessing unique features have advantages in this regard. For example, paclitaxel is widely used as an anticancer drug. Gibson et al ${ }^{58}$ first covalently combined 2-nm AuNPs with paclitaxel; thus, approximately 70 paclitaxel particles could be attached to a single NP. This conjugation method maintained the core size and shape of AuNPs and their high drug-loading capacity. Cisplatin has been clinically used as an anticancer drug and a radiosensitizer. Cisplatin can induce cell-cycle arrest, inhibit DNA replication, and trigger apoptosis as well as Auger electron generation owing to the high $Z$ number of platinum. Nonetheless, the use of free cisplatin in chemoradiotherapy is limited owing to its toxicity, necessitating hybrid NPs to enhance radiosensitivity and reduce cytotoxicity. Zheng and Sanche ${ }^{59}$ conjugated cisplatin and AuNPs to plasmid DNA to form a complex to examine radioactivity enhancement. The double-strand breaks in DNA increased radioactivity by a factor of 3 when only one AuNP was involved compared with a factor of 7.5 when two cisplatin molecules and one AuNP were conjugated in a complex. According to a recent study by Cui et al, ${ }^{60}$ AuNPs improve the treatment of triplenegative breast cancer. AuNPs ( $0.5 \mathrm{mg}$ of gold per tumor) exert an effect equivalent to that of three cisplatin doses at a $25 \%$ effective concentration ( $\mathrm{IC}_{25} ; 4 \mathrm{mg} / \mathrm{kg}$ ), indicating high toxicity toward normal cells. More recently, Davidi et a ${ }^{61}$ examined head and neck cancer both in vitro and in vivo, and AuNPs were more effective in radiotherapy compared with free cisplatin.

\section{Advanced drug delivery systems for radiosensitization Mesoporous drug delivery system}

Mesoporous materials have been utilized for effective controlled release and drug delivery; their high pore volume and large surface area guarantee a high drug-loading capacity, whereas the ordered porous network enables the fine control of drug loading and release kinetics. ${ }^{62}$ Fan et a ${ }^{63}$ synthesized a novel rattle-structured upconversion core/porous silica shell nanotheranostic system loaded with cisplatin and observed a significant improvement in therapeutic efficacy compared with that of free cisplatin. A dual-mode magnetic resonance imaging (MRI)/upconversion signal was observed for synergistic drug tracing and monitoring. They later modified this rattle structure into a 50-nm nucleus-targeting upconversion core/mesoporous silica nanotheranostic system ${ }^{64}$ and demonstrated the direct delivery of the radiosensitizing drug mitomycin C into the nucleus, where ROS generated by ionizing radiation damaged most of the DNA within cancer cells. With the aim of targeting hypoxia to enhance the effects of radiation, they next fabricated the hypoxiaactivated prodrug tirapazamine with UCNPs as the core and mesoporous silica serving as the shell. ${ }^{65}$ Hypoxia-induced tirapazamine activation was amplified by the UCNP core, thereby enhancing the efficacy of radiotherapy. In addition to silica, Song et a ${ }^{66}$ creatively synthesized an "all-in-one" hollow tantalum oxide structure by a one-pot method. The mesoporous structure had the capacity to load an anticancer drug (7-ethyl-10-hydroxycamptothecin, SN-38), while achieving radiosensitization owing to its intrinsic high- $Z$ ( $Z=73$ ). Moreover, these NPs can bind to metal ions, such as $\mathrm{Fe}^{3+}$ and ${ }^{99 \mathrm{~m}} \mathrm{Tc}^{4+}$, by a facile method, resulting in functionalization for MRI and single-photon emission CT imaging. Based on a similar idea, doxorubicin-loaded $\mathrm{TaO}_{x}$ NPs were fabricated; $\mathrm{mTa}_{2} \mathrm{O}_{5}$ NPs significantly inhibited tumor growth compared with single modality treatments. Furthermore, this approach achieved the therapeutic goal under a safe dosage, which is lethal when applied in free doxorubicin. ${ }^{67}$

\section{Liposomes as drug carriers}

As classic carriers for drug delivery, liposomes can entrap drugs within the phospholipid bilayer structure and deliver them specifically to tumor sites by the EPR effect. Numerous studies have made significant progress in optimizing the rate 
of encapsulated drug release, while retaining bioactivity. Researchers have made efforts to prolong the circulation half-life to reduce rapid clearance by the mononuclear phagocyte system. ${ }^{68-70}$ PEGylated liposomes have been identified as general carriers for drug delivery, with high efficacy in synergistic chemoradiotherapy, prompting the first clinical trial of PEG liposomes as carriers of doxorubicin for Kaposi's sarcoma in patients with AIDS. ${ }^{71}$ Recently, liposomes were utilized in hypoxia-targeted chemotherapy. Liu et al ${ }^{72}$ developed liposome-cholesterol-based NPs conjugated with the radiosensitizer nitroimidazole by a hydrolyzable ester bond; doxorubicin was next loaded as an anticancer drug. These NPs penetrated the blood-brain barrier to accumulate in malignant gliomas owing to the lipophilic surface, and therein converted hydrophobic nitroimidazoles into hydrophilic aminoimidazole under hypoxic conditions to achieve enhanced chemoradiotherapeutic effects of doxorubicin. To further improve the therapeutic efficacy, they transported an siRNA into the glioma via the same liposomes and achieved higher cellular uptake by taking advantage of the positive charges induced by hypoxia and a low $\mathrm{pH}^{73}$ Different from these studies, Zhang et $\mathrm{al}^{74}$ synthesized a novel cisplatin (IV)prodrug-conjugated phospholipid, while encapsulating catalase as an internal $\mathrm{O}_{2}$ generator that degrades $\mathrm{H}_{2} \mathrm{O}_{2}$ in the TME. The bilayered liposomes had a protective effect to maintain protease activity. In a proteinase $\mathrm{K}$ digestion assay aimed at simulating the physiological environment, $81 \%$ of the initial catalytic activity was retained compared with $35 \%$ without protection by liposomes.

\section{HIF-I-targeted therapy}

HIF-1 is a two-heterodimeric protein that is upregulated in response to the dysfunction of the $\mathrm{O}_{2}$-dependent degradation pathway, leading to the activation of multiple oncogenes, such as $V E G F, M D R 1, M M P 2$, and $U P A R$, as well as malignant biological properties, such as angiogenesis, proliferation, and invasion/metastasis. ${ }^{4,75,76}$ Recent studies have demonstrated that HIF-1 mainly contributes to radioresistance by enhancing vascular reconstruction postiradiation. ${ }^{75,77,78}$

HIF-targeted drugs can be assigned to two main categories: inhibitors of the HIF-1 signaling pathway (RAF kinase, mTOR, and EGFR) and inhibitors of HIF-1 activation (topoisomerase I and HSP90). However, the adverse effects of single drugs limit their efficacy in therapeutic applications, prompting the development of NP-conjugated nanomedicines. For example, the novel nano-conjugated drug CRLX101 $1{ }^{79}$ containing the topoisomerase I inhibitor camptothecin as the payload and modified with betacyclodextrin and PEG into NPs (20-50 nm in diameter), achieved satisfactory tumor accumulation by the EPR effect and sustained intracellular drug release, ${ }^{80,81}$ thus significantly reducing side effects and increasing bioavailability. Although clinical studies have not demonstrated a benefit of CRLX101 in patients with renal cancer with respect to overall survival, ${ }^{79}$ Rey et $\mathrm{a}^{16}$ suggested that additional clinical trials considering HIF- $\alpha$ and intratumoral hypoxia as important factors should be designed to determine the benefits of HIF-1 and downstream inhibitors.

In addition to nano-conjugated drugs, recent efforts have been made to use nanomaterials as smart delivery systems to transport HIF-1-targeting siRNAs into hypoxic tumor cells for gene therapy. For example, Chen et al ${ }^{82}$ improved drug permeability by preparing a novel biodegradable d- $\alpha$-tocopheryl PEG 1,000 succinate as delivery system to transport siRNAs into nasopharyngeal carcinoma cells, thus inhibiting HIF-1 in vitro and in vivo. In another study by Zhao et al, ${ }^{83}$ anionic siRNA was attached to the surface of a cationic $\varepsilon$-polylysine co-polymer to form a stable conjugate, and gemcitabine was further encapsulated into the hydrophilic core and modified with PEG for the codelivery of dual anticancer drugs; this resulted in an excellent therapeutic efficacy to inhibit tumor metastasis in an orthotopic tumor model. Nevertheless, the versatility of nanomaterial-based drug delivery systems has not been fully investigated to optimize HIF-1-targeted therapies that function synergistically with radiotherapy. The codelivery of HIF-targeting drugs, a high- $Z$ element-based radiosensitizer, and a smart hypoxia/ pH-responsive drug-release system for selective HIF-1 inhibition should be studied more in the future.

\section{X-ray- or NIR-responsive NPs for radiosensitization}

As a novel approach to the treatment of hypoxic tumors in a time-dependent and site-specific manner, an external stimulus-responsive therapeutic agent may be beneficial for clinical applications aimed at maximizing radiosensitivity according to pharmacological parameters. Recently, NIR light and/or X-rays have been used to ensure controlled drug release and synergistic thermo/photodynamic/radiotherapy. Various nanoplatforms serve as amplifiers to increase therapeutic efficacy and minimize adverse effects.

\section{NIR-responsive NPs for stimulus- responsive activation or photothermal therapy}

Photothermal agents can absorb NIR light at certain wavelengths and convert the NIR light energy into heat to produce local hyperthermia, resulting in tumor necrosis and 
intracellular protein denaturation. Moreover, photothermal therapy can boost blood flow to tumors to partially relieve hypoxia, thus overcoming hypoxia-related radioresistance. ${ }^{84,85}$ Gold $(Z=79)$ is a representative high- $Z$ element often chosen for radiosensitization. Recent studies have investigated its ability to absorb NIR light as a photothermal agent. For example, Ma et $\mathrm{al}^{50}$ developed novel hollow spike-like gold nanostructures in a facile galvanic replacement reaction and tested their efficacy in experimental groups subjected to thermo-, radio-, and thermoradiotherapy. The thermoradiotherapy group exhibited superior tumor growth inhibition $(92.2 \%)$ in vivo compared with that in the AuNP-mediated single-modality radiotherapy group $(29.8 \%)$ or the thermotherapy group, indicating that hyperthermia effectively enhances the effect of radiosensitization on hypoxic cancer cells resistant to ionizing radiation via a synergistic effect. In another study, Dou et $\mathrm{al}^{86}$ constructed a multifunctional theranostic system by fabricating Prussian blue NPs and AuNPs in a core-shell system; the Prussian blue NPs served as a magnetic resonance contrast agent and photothermal agent, whereas the AuNPs acted as radiosensitizers and CT contrast agents, exerting remarkably effective therapeutic effects compared with those of the individual therapeutic modalities. These results prompted researchers to further explore advanced materials for the development of a thermotherapeutic nanoplatform.

Unlike the abovementioned AuNPs, UCNPs convert less optical energy into heat owing to the low extinction coefficient of lanthanide ions; thus, plasmonic NPs are commonly integrated into such nanoplatforms. Moreover, upconverted luminescence can offer a unique feature for imaging-guided photothermal therapy and/or radiotherapy. As an example, Xiao et $\mathrm{l}^{87}$ creatively synthesized a core-satellite nanostructure by attaching ultrasmall CuS NPs onto the surface of UCNPs@ $\mathrm{SiO}_{2}$ and performed trimodal CT/MRI/upconversion photoluminescence imaging for effective diagnosis; the combination of photothermal therapy and radiotherapy completely eradicated the tumor and prevented its recurrence for up to 120 days. As part of a preclinical investigation, Liu et al ${ }^{88}$ doped $\mathrm{CuS}$ with the ${ }^{131}$ I isotope to evaluate the efficacy of thermo/radiotherapy for IBRT to treat subcutaneous and metastatic lesions; remarkably, they discovered that $\mathrm{CuS} /{ }^{131} \mathrm{I}$ NPs accumulated in primary solid tumors and migrated to (and were retained within) nearby sentinel lymph node, producing therapeutic effects orthotopically and inhibiting the formation of metastatic lesions. Zou et $\mathrm{al}^{89}$ summarized these findings as follows: photothermal-therapyrelated metastatic inhibition can be attributed to 1) mild hyperthermia-induced cell eradication as well as metastasisrelated factors, 2) proper planning of timing and location by real-time imaging, and 3) synergistic effects of radiotherapy and chemotherapy. Although it is believed that NIR light has limited penetration depth and causes hyperthermia in normal tissues, more efforts should be devoted to optimize the synergistic therapeutic effects, and thermo/radiotherapy still holds great promise for improving cancer therapies.

\section{X-ray/NIR-responsive NPs for hypoxia radiosensitization}

The controlled release and activation of drugs or prodrugs are highly appealing for clinical applications by enabling drug delivery to specific tumor sites and drug activation only during irradiation; thus, concomitant damage to surrounding normal tissues is minimized. X-rays and NIR have been explored as major agents for such drug delivery systems. First, X-rays have beneficial characteristics for prompt and selective drug activation, including 1) deep penetration and precise localization, enabling drug release in a preplanned, spatially dependent manner, 2) responsive drug release in a controlled manner via the manipulation of the radiation dose, 3) X-ray-induced damage to the tumor itself. ${ }^{90}$ For example, the most commonly loaded drugs for radiosensitization are nitric oxide $\left(\mathrm{NO}_{\mathrm{x}}\right)$-based prodrugs, serving as X-ray sensitizers by promoting tumor cell apoptosis, enhancing X-ray-induced DNA damage, and exerting a "by-stander" effect. ${ }^{91,92}$ Another study has shown that these effects are associated with the activation of the tumor suppressor gene p53. ${ }^{91}$

Fan et $\mathrm{al}^{93}$ developed a novel nanostructure for the transport and selective activation of nitro group-based drugs; in particular, they showed that UCNPs together with $S$-nitrosothiol-grafted mesoporous silica (PEG-USMSsSNO) achieve hypoxic radiosensitization on demand via $\mathrm{X}$-ray dose-dependent NO release. In a more recent study, Liu et $\mathrm{al}^{94}$ grafted nitroimidazole onto AuNPs by a facile method and found that lower X-ray doses (0.71 Gy) are required in single-modality radiotherapy to achieve the same therapeutic effects. In another study, Guo et al ${ }^{95}$ creatively fabricated $\mathrm{BiOI} @ \mathrm{Bi}_{2} \mathrm{~S}_{3} @ \mathrm{BSA}$ semiconductor heterojunction NPs for combined radio/photodynamic/photothermal cancer therapy and multimodal CT/photoacoustics (CT/PA); the all-in-one nanoplatform elevated the synergistic radio/ photodynamic/photothermal therapeutic efficacy, as observed for the CT/PA combination, which utilizes the advantages of both diagnostic tools, including a higher sensitivity, higher spatial resolution, and deeper penetration. 
In clinical settings, the major factor limiting radiation dose is adverse effects. Given that ionizing radiation inevitably escapes to surrounding healthy tissues, it is important to control adverse effects as well as enhance therapeutic effects. Du et a ${ }^{96}$ developed a novel nanoscaled radiosensitizer based on poly(vinylpyrrolidone)- and selenocysteine-modified $\mathrm{Bi}_{2} \mathrm{Se}_{3} \mathrm{NPs}\left(\mathrm{PVP}-\mathrm{Bi}_{2} \mathrm{Se}_{3} @ \mathrm{Sec}\right.$ NPs); the bismuth core, as a high- $Z$ element, can amplify the X-ray dose, while absorbing NIR light to generate heat for photothermal ablation and high photothermal conversion efficiency. Of note, selenium released from the decomposing NPs exerts considerable protective effects by reducing excess free radicals and eliminating the concomitant toxicity. These findings demonstrate a heuristic approach to the development of new platforms for comprehensively increasing therapeutic efficacy.

\section{Conclusion and future perspectives}

In this review, we comprehensively summarized recent progress in improving radiotherapy efficacy by targeting hypoxia. Based on the correlation between hypoxia and radioresistance, multiple innovative materials have been developed for hypoxic tumor treatment, including 1) a number of materials that act as carriers to deliver oxygen directly into hypoxic tumor regions to relieve hypoxia, 2) NPs containing high- $Z$ elements to deposit radiation energy within tumors and amplify X-ray-induced damage, 3) intelligent drug delivery systems to load multiple nanomaterials or drugs, such as hypoxia-activated prodrugs or high- $Z$ elements, 4) X-ray/NIR-responsive nanomaterials for selective drug activation or hyperthermia therapy for synergistic thermo-radiotherapy.

Nanomaterials aimed at increasing oxygen levels in tumors offer a practical method for modulating the TME. Although in vivo and in vitro studies have demonstrated improvements in the effects of radiotherapy, it is important to further clarify the spatial relationship between the distribution of oxygen molecules and hypoxic tumor regions as well as to optimize the time post-reoxygenation for radiotherapy. In this regard, radiolabeled NPs are promising for further studies owing to their stable half-life and effectiveness.

High- $Z$ elements, such as gold or bismuth, can improve radiosensitivity. Recent studies have focused on modulating the shape and size of NPs to further improve the radiosensitizing ability. It is also critical to increase the intake of these NPs by tumor cells as well as to reduce nonspecific uptake and accelerate renal clearance to avoid long-term body retention.
X-ray/NIR-responsive NPs enable selective photothermal therapy or drug activation and demonstrate efficacy in improving radiotherapy in vivo. In addition to the antitumor efficacy caused by hyperthermia, boosted blood flow promotes reoxygenation to modulate the tumor environment. However, the limited penetration depth of NIR light is a major concern for deep tumor ablation and synergistic thermal-radiotherapy, and further studies are needed to determine the optimal combination of two therapies. Nevertheless, the integration of radiotherapy and photothermal therapy still holds great promise for future clinical translation.

\section{Acknowledgement}

This paper is supported by the National Key Research and Development Program of China (Grant No. 2017YFA0205 200), the National Natural Science Foundation of China (Grant Nos. 81571785, 81771957, 81502642, 81227901, 81527805, 61671449 and 81501540), the Natural Science Foundation of Guangdong Province, China (Grant Nos. 2016A030311055, 2016A030313770, 2015A030310108), the Chinese Academy of Sciences (Grant No. GJJSTD20170004), and the State Key Laboratory of Management and Control for Complex Systems, Institute of Automation, Chinese Academy of Sciences (Grant No. 20180111).

\section{Disclosure}

The authors report no conflicts of interest in this work.

\section{References}

1. Vaupel P, Kelleher DK, Höckel M. Oxygen status of malignant tumors: pathogenesis of hypoxia and significance for tumor therapy. Semin Oncol. 2001;28(2 Suppl 8):29-35.

2. Vaupel P, Schlenger K, Knoop C, Höckel M. Oxygenation of human tumors: evaluation of tissue oxygen distribution in breast cancers by computerized $\mathrm{O}_{2}$ tension measurements. Cancer Res. 1991;51(12): 3316-3322.

3. Hockel M, Schlenger K, Aral B, et al. Association between tumor hypoxia and malignant progression in advanced cancer of the uterine cervix. Cancer Res. 1996;56(19):4509-4515.

4. Semenza GL. Targeting HIF-1 for cancer therapy. Nat Rev Cancer. 2003; 3(10):721-732.

5. Minchinton AI, Tannock IF. Drug penetration in solid tumours. Nat Rev Cancer. 2006;6(8):583-592.

6. Rey S, Schito L, Koritzinsky M, Wouters BG. Molecular targeting of hypoxia in radiotherapy. Adv Drug Deliv Rev. 2017;109:45-62.

7. Yao Y, Zhang M, Liu T, et al. Perfluorocarbon-encapsulated PLGAPEG emulsions as enhancement agents for highly efficient reoxygenation to cell and organism. ACS Appl Mater Interfaces. 2015; 7(33):18369-18378.

8. Brown JM, Wilson WR. Exploiting tumour hypoxia in cancer treatment Nat Rev Cancer. 2004;4(6):437-447.

9. Carmeliet P, Jain RK. Molecular mechanisms and clinical applications of angiogenesis. Nature. 2011;473(7347):298-307. 
10. Horsman MR, Mortensen LS, Petersen JB, Busk M, Overgaard J. Imaging hypoxia to improve radiotherapy outcome. Nat Rev Clin Oncol. 2012; 9(12):674-687.

11. Wilson WR, Hay MP. Targeting hypoxia in cancer therapy. Nat Rev Cancer. 2011;11(6):393-410.

12. Teicher BA, Rose CM. Oxygen-carrying perfluorochemical emulsion as an adjuvant to radiation therapy in mice. Cancer Res. 1984;44(10): 4285-4288.

13. Teicher BA, Rose CM. Perfluorochemical emulsions can increase tumor radiosensitivity. Science. 1984;223(4639):934-936.

14. Hartzler GO, Rutherford BD, Mcconahay DR, Johnson WL, Giorgi LV. "High-risk" percutaneous transluminal coronary angioplasty. Am J Cardiol. 1988;61(14):33G-37G.

15. Teicher BA, Holden SA, Ara G, Dupuis NP, Goff D. Restoration of tumor oxygenation after cytotoxic therapy by a perflubron emulsion/ carbogen breathing. Cancer J Sci Am. 1995;1(1):43-48.

16. Feldman LA, Fabre MS, Grasso C, et al. Perfluorocarbon emulsions radiosensitise brain tumors in carbogen breathing mice with orthotopic GL261 gliomas. PLoS ONE. 2017;12(9):e0184250.

17. Wouters BG, Brown JM. Cells at intermediate oxygen levels can be more important than the "hypoxic fraction" in determining tumor response to fractionated radiotherapy. Radiat Res. 1997;147(5):541-550.

18. Lee HY, Kim HW, Lee JH, Oh SH. Controlling oxygen release from hollow microparticles for prolonged cell survival under hypoxic environment. Biomaterials. 2015;53:583-591.

19. Cheng Y, Cheng H, Jiang C, et al. Perfluorocarbon nanoparticles enhance reactive oxygen levels and tumour growth inhibition in photodynamic therapy. Nat Commun. 2015;6:8785.

20. Song G, Liang C, Yi X, et al. Perfluorocarbon-loaded hollow $\mathrm{Bi}_{2} \mathrm{Se}_{3}$ nanoparticles for timely supply of oxygen under near-infrared light to enhance the radiotherapy of cancer. Adv Mater. 2016;28(14): 2716-2723.

21. Song G, Ji C, Liang $\mathrm{C}$, et al. $\mathrm{TaO}_{\mathrm{x}}$ decorated perfluorocarbon nanodroplets as oxygen reservoirs to overcome tumor hypoxia and enhance cancer radiotherapy. Biomaterials. 2017;112:257-263.

22. Porter T, Kricsfeld D, Cheatham S, Li S. Effect of blood and microbubble oxygen and nitrogen content on perfluorocarbon-filled dextrose albumin microbubble size and efficacy: in vitro and in vivo studies. $J$ Am Soc Echocardiogr. 1998;11(5):421-425.

23. Swanson EJ, Mohan V, Kheir J, Borden MA. Phospholipid-stabilized microbubble foam for injectable oxygen delivery. Langmuir. 2010; 26(20):15726-15729.

24. Cavalli R, Bisazza A, Rolfo A, et al. Ultrasound-mediated oxygen delivery from chitosan nanobubbles. Int J Pharm. 2009;378(1-2):215-217.

25. Eisenbrey JR, Albala L, Kramer MR, et al. Development of an ultrasound sensitive oxygen carrier for oxygen delivery to hypoxic tissue. Int J Pharm. 2015;478(1):361-367.

26. Kwan JJ, Kaya M, Borden MA, Dayton PA. Theranostic oxygen delivery using ultrasound and microbubbles. Theranostics. 2012;2(12): 1174-1184.

27. Song X, Feng L, Liang C, Yang K, Liu Z. Ultrasound triggered tumor oxygenation with oxygen-shuttle nanoperfluorocarbon to overcome hypoxia-associated resistance in cancer therapies. Nano Lett. 2016;16(10):6145-6153.

28. Wang P, Li X, Yao C, et al. Orthogonal near-infrared upconversion co-regulated site-specific $\mathrm{O}_{2}$ delivery and photodynamic therapy for hypoxia tumor by using red blood cell microcarriers. Biomaterials. 2017;125:90-100

29. Ishida T, Masuda K, Ichikawa T, et al. Accelerated clearance of a second injection of PEGylated liposomes in mice. Int J Pharm. 2003;255(1-2): 167-174.

30. Gao M, Liang C, Song X, et al. Erythrocyte-membrane-enveloped perfluorocarbon as nanoscale artificial red blood cells to relieve tumor hypoxia and enhance cancer radiotherapy. Adv Mater. 2017; 29(35):1701429.

31. Ren H, Liu J, Li Y, et al. Oxygen self-enriched nanoparticles functionalized with erythrocyte membranes for long circulation and enhanced phototherapy. Acta Biomater. 2017;59:269-282.
32. Kuang Y, Balakrishnan K, Gandhi V, Peng X. Hydrogen peroxide inducible DNA cross-linking agents: targeted anticancer prodrugs. J Am Chem Soc. 2011;133(48):19278-19281.

33. Abbasi AZ, Gordijo CR, Amini MA, et al. Hybrid manganese dioxide nanoparticles potentiate radiation therapy by modulating tumor hypoxia. Cancer Res. 2016;76(22):6643-6656.

34. Cho MH, Choi E, Kim S, Goh S, Choi Y. Redox-responsive manganese dioxide nanoparticles for enhanced MR imaging and radiotherapy of lung cancer. Front Chem. 2017;5:109.

35. Huang F, Xia F. A smart photodynamic therapy strategy: depletion of glutathione in cancer cells. Sci Bull. 2017;62(5):312-313.

36. Tian L, Chen Q, Yi X, et al. Albumin-templated manganese dioxide nanoparticles for enhanced radioisotope therapy. Small. 2017; 13(25):1700640.

37. Fan $\mathrm{W}, \mathrm{Bu} \mathrm{W}$, Shen $\mathrm{B}$, et al. Intelligent $\mathrm{MnO}_{2}$ nanosheets anchored with upconversion nanoprobes for concurrent $\mathrm{pH}-/ \mathrm{H}_{2} \mathrm{O}_{2}$-responsive UCL imaging and oxygen-elevated synergetic therapy. Adv Mater. 2015;2 $7(28): 4155-4161$.

38. Yang G, Zhang R, Liang C, et al. Manganese dioxide coated WS2@, $\mathrm{Fe}_{3} \mathrm{O}_{4} / \mathrm{SSiO}_{2}$ nanocomposites for $\mathrm{pH}$-responsive $\mathrm{MR}$ imaging and oxygen-elevated synergetic therapy. Small. 2018;14(2)

39. Yi X, Chen L, Zhong X, et al. Core-shell $\mathrm{Au} @ \mathrm{MnO}_{2}$ nanoparticles for enhanced radiotherapy via improving the tumor oxygenation. Nano Res. 2016;9(11):3267-3278.

40. Liu J, Chen Q, Zhu W, et al. Nanoscale-coordination-polymer-shelled manganese dioxide composite nanoparticles: a multistage redox/pH/ $\mathrm{H}_{2} \mathrm{O}_{2}$-responsive cancer theranostic nanoplatform. Adv Funct Mater. 2017;27(10): 1605926 .

41. Adams GE. Hypoxic cell sensitizers for radiotherapy. Int J Radiat Oncol Biol Phys. 1978;4(1-2):135-141.

42. Cui L, Tse K, Zahedi P, et al. Hypoxia and cellular localization influence the radiosensitizing effect of gold nanoparticles (AuNPs) in breast cancer cells. Radiat Res. 2014;182(5):475-488.

43. Yen HJ, Hsu SH, Tsai CL. Cytotoxicity and immunological response of gold and silver nanoparticles of different sizes. Small. 2009;5(13):1553-1561.

44. Bartneck M, Keul HA, Singh S, et al. Rapid uptake of gold nanorods by primary human blood phagocytes and immunomodulatory effects of surface chemistry. ACS Nano. 2010;4(6):3073-3086.

45. Taylor U, Klein S, Petersen S, et al. Nonendosomal cellular uptake of ligand-free, positively charged gold nanoparticles. Cytometry A. 2010; 77(5):439-446.

46. Arvizo RR, Bhattacharyya S, Kudgus RA, et al. Intrinsic therapeutic applications of noble metal nanoparticles: past, present and future. Chem Soc Rev. 2012;41(7):2943-2970.

47. Jain S, Coulter JA, Butterworth KT, et al. Gold nanoparticle cellular uptake, toxicity and radiosensitisation in hypoxic conditions. Radiother Oncol. 2014;110(2):342-347.

48. van Haute D, Liu AT, Berlin JM. Coating metal nanoparticle surfaces with small organic molecules can reduce nonspecific cell uptake. ACS Nano. 2018;12(1):117-127.

49. Shen J, Kim $\mathrm{HC}, \mathrm{Mu} \mathrm{C}$, et al. Multifunctional gold nanorods for siRNA gene silencing and photothermal therapy. Adv Healthc Mater. 2014;3(10):1629-1637.

50. Ma N, Liu P, He N, et al. Action of gold nanospikes-based nanoradiosensitizers: cellular internalization, radiotherapy, and autophagy. ACS Appl Mater Interfaces. 2017;9(37):31526-31542.

51. Liang G, Jin X, Zhang S, Xing D. RGD peptide-modified fluorescent gold nanoclusters as highly efficient tumor-targeted radiotherapy sensitizers. Biomaterials. 2017;144:95-104.

52. Bhattarai SR, Derry PJ, AzizK, et al. Gold nanotriangles: scale up and X-ray radiosensitization effects in mice. Nanoscale. 2017;9(16):5085-5093.

53. Dou Y, Guo Y, Li X, et al. Size-tuning ionization to optimize gold nanoparticles for simultaneous enhanced CT imaging and radiotherapy. ACS Nano. 2016;10(2):2536-2548.

54. Ma N, Wu FG, Zhang X, et al. Shape-dependent radiosensitization effect of gold nanostructures in cancer radiotherapy: comparison of gold nanoparticles, nanospikes, and nanorods. ACS Appl Mater Interfaces. 2017;9(15):13037-13048. 
55. Sauer R, Becker H, Hohenberger W, et al. Preoperative versus postoperative chemoradiotherapy for rectal cancer. NEngl J Med. 2004;351(17): 1731-1740.

56. Sebag-Montefiore D, Stephens RJ, Steele R, et al. Preoperative radiotherapy versus selective postoperative chemoradiotherapy in patients with rectal cancer (MRC CR07 and NCIC-CTG C016): a multicentre, randomised trial. Lancet. 2009;373(9666):811-820.

57. Bosset JF, Collette L, Calais G, et al. Chemotherapy with preoperative radiotherapy in rectal cancer. $N$ Engl $J$ Med. 2006;355(11): 1114-1123.

58. Gibson JD, Khanal BP, Zubarev ER. Paclitaxel-functionalized gold nanoparticles. J Am Chem Soc. 2007;129(37):11653-11661.

59. Zheng Y, Sanche L. Gold nanoparticles enhance DNA damage induced by anti-cancer drugs and radiation. Radiat Res. 2009;172(1):114-119.

60. Cui L, Her S, Dunne M, et al. Significant radiation enhancement effects by gold nanoparticles in combination with cisplatin in triple negative breast cancer cells and tumor xenografts. Radiat Res. 2017;187(2):147-160.

61. Davidi ES, Dreifuss T, Motiei M, et al. Cisplatin-conjugated gold nanoparticles as a theranostic agent for head and neck cancer. Head Neck. 2018;40(1):70-78.

62. Vallet-Regí M, Balas F, Arcos D. Mesoporous materials for drug delivery. Angew Chem Int Ed Engl. 2007;46(40):7548-7558.

63. Fan W, Shen B, Bu W, et al. Rattle-structured multifunctional nanotheranostics for synergetic chemo-/radiotherapy and simultaneous magnetic/luminescent dual-mode imaging. J Am Chem Soc. 2013;135(17): 6494-6503.

64. Fan W, Shen B, Bu W, et al. Design of an intelligent sub-50 nm nucleartargeting nanotheranostic system for imaging guided intranuclear radiosensitization. Chem Sci. 2015;6(3):1747-1753.

65. Liu Y, Liu Y, Bu W, et al. Radiation-/hypoxia-induced solid tumor metastasis and regrowth inhibited by hypoxia-specific upconversion nanoradiosensitizer. Biomaterials. 2015;49:1-8.

66. Song G, Chao Y, Chen Y, et al. All-in-one theranostic nanoplatform based on hollow $\mathrm{TaO}_{\mathrm{x}}$ for chelator-free labeling imaging, drug delivery, and synergistically enhanced radiotherapy. Adv Funct Mater. 2016; 26(45):8243-8254

67. Chen Y, Song G, Dong Z, et al. Drug-loaded mesoporous tantalum oxide nanoparticles for enhanced synergetic chemoradiotherapy with reduced systemic toxicity. Small. 2017;13(8):1602869.

68. Wicki A, Witzigmann D, Balasubramanian V, Huwyler J. Nanomedicine in cancer therapy: challenges, opportunities, and clinical applications. J Control Rel. 2015;200:138-157.

69. Allen TM, Cullis PR. Liposomal drug delivery systems: from concept to clinical applications. Adv Drug Deliv Rev. 2013;65(1):36-48.

70. Zhu Y, Liao L. Applications of nanoparticles for anticancer drug delivery: a review. J Nanosci Nanotechnol. 2015;15(7):4753-4773.

71. Masood R, Husain SR, Rahman A, Gill P. Potentiation of cytotoxicity of Kaposi's sarcoma related to immunodeficiency syndrome (AIDS) by liposome-encapsulated doxorubicin. AIDS Res Hum Retroviruses. 1993;9(8):741-746.

72. Liu H, Xie Y, Zhang Y, et al. Development of a hypoxia-triggered and hypoxic radiosensitized liposome as a doxorubicin carrier to promote synergetic chemo-/radio-therapy for glioma. Biomaterials. 2017;121: $130-143$.

73. Liu HM, Zhang YF, Xie YD, et al. Hypoxia-responsive ionizable liposome delivery siRNA for glioma therapy. Int J Nanomedicine. 2017; 12:1065-1083.

74. Zhang R, Song X, Liang C, et al. Catalase-loaded cisplatin-prodrugconstructed liposomes to overcome tumor hypoxia for enhanced chemoradiotherapy of cancer. Biomaterials. 2017;138:13-21.

75. Schito L, Semenza GL. Hypoxia-inducible factors: master regulators of cancer progression. Trends Cancer. 2016;2(12):758-770.

76. Rey S, Schito L, Wouters BG, Eliasof S, Kerbel RS. Targeting hypoxiainducible factors for antiangiogenic cancer therapy. Trends Cancer. 2017;3(7):529-541.

77. Dewhirst MW, Cao Y, Moeller B. Cycling hypoxia and free radicals regulate angiogenesis and radiotherapy response. Nat Rev Cancer. 2008; $8(6): 425-437$.
78. Moeller BJ, Cao Y, Li CY, Dewhirst MW. Radiation activates HIF-1 to regulate vascular radiosensitivity in tumors: role of reoxygenation, free radicals, and stress granules. Cancer Cell. 2004;5(5):429-441.

79. Voss MH, Hussain A, Vogelzang N, et al. A randomized phase II trial of CRLX101 in combination with bevacizumab versus standard of care in patients with advanced renal cell carcinoma. Ann Oncol. 2017 ; 28(11):2754-2760.

80. Maeda H. Toward a full understanding of the EPR effect in primary and metastatic tumors as well as issues related to its heterogeneity. $A d v$ Drug Deliv Rev. 2015;91:3-6.

81. Clark AJ, Wiley DT, Zuckerman JE, et al. CRLX101 nanoparticles localize in human tumors and not in adjacent, nonneoplastic tissue after intravenous dosing. Proc Natl Acad Sci USA. 2016;113(14): 3850-3854.

82. Chen Y, Xu G, Zheng Y, et al. Nanoformulation of D- $\alpha$-tocopheryl polyethylene glycol 1000 succinate-b-poly( $\varepsilon$-caprolactone-ran-glycolide) diblock copolymer for siRNA targeting HIF-1 $\alpha$ for nasopharyngeal carcinoma therapy. Int J Nanomed. 2015;10:1375-1386.

83. Zhao X, Li F, Li Y, et al. Co-delivery of HIF $1 \alpha$ siRNA and gemcitabine via biocompatible lipid-polymer hybrid nanoparticles for effective treatment of pancreatic cancer. Biomaterials. 2015;46:13-25.

84. Liu JN, Bu W, Shi J. Chemical design and synthesis of functionalized probes for imaging and treating tumor hypoxia. Chem Rev. 2017; 117(9):6160-6224.

85. Song G, Cheng L, Chao Y, Yang K, Liu Z. Emerging nanotechnology and advanced materials for cancer radiation therapy. Adv Mater. 2017;29(32):1700996

86. Dou Y, Li X, Yang W, et al. PB@Au core-satellite multifunctional nanotheranostics for magnetic resonance and computed tomography imaging in vivo and synergetic photothermal and radiosensitive therapy. ACS Appl Mater Interfaces. 2017;9(2):1263-1272.

87. Xiao Q, Zheng X, Bu W, et al. A core/satellite multifunctional nanotheranostic for in vivo imaging and tumor eradication by radiation/ photothermal synergistic therapy. J Am Chem Soc. 2013;135(35): 13041-13048.

88. Yi X, Yang K, Liang C, et al. Imaging-guided combined photothermal and radiotherapy to treat subcutaneous and metastatic tumors using iodine-131-doped copper sulfide nanoparticles. Adv Funct Mater. 2015; 25(29):4689-4699.

89. Zou L, Wang H, He B, et al. Current approaches of photothermal therapy in treating cancer metastasis with nanotherapeutics. Theranostics. 2016;6(6):762-772.

90. Ma N, Xu H, An L, et al. Radiation-sensitive diselenide block copolymer micellar aggregates: toward the combination of radiotherapy and chemotherapy. Langmuir. 2011;27(10):5874-5878.

91. Cook T, Wang Z, Alber S, et al. Nitric oxide and ionizing radiation synergistically promote apoptosis and growth inhibition of cancer by activating p53. Cancer Res. 2004;64(21):8015-8021.

92. Kim J, Yung BC, Kim WJ, Chen X. Combination of nitric oxide and drug delivery systems: tools for overcoming drug resistance in chemotherapy. J Control Rel. 2017;263(SI):223-230.

93. Fan W, Bu W, Zhang Z, et al. X-ray radiation-controlled NO-release for on-demand depth-independent hypoxic radiosensitization. Angew Chem Int Ed Engl. 2015;54(47):14026-14030.

94. Liu F, Lou J, Hristov D. X-ray responsive nanoparticles with triggered release of nitrite, a precursor of reactive nitrogen species, for enhanced cancer radiosensitization. Nanoscale. 2017;9(38):14627-14634.

95. Guo Z, Zhu S, Yong Y, et al. Synthesis of BSA-coated BiOI@Bi, S semiconductor heterojunction nanoparticles and their applications for radio/photodynamic/photothermal synergistic therapy of tumor. Adv Mater. 2017;29(44):1704136.

96. Du J, Gu Z, ss Yan L, et al. Poly(vinylpyrollidone)- and selenocysteinemodified $\mathrm{Bi}_{2} \mathrm{Se}_{3}$ nanoparticles enhance radiotherapy efficacy in tumors and promote radioprotection in normal tissues. Adv Mater. 2017; 29(34):1701268. 


\section{Publish your work in this journal}

The International Journal of Nanomedicine is an international, peerreviewed journal focusing on the application of nanotechnology in diagnostics, therapeutics, and drug delivery systems throughout the biomedical field. This journal is indexed on PubMed Central, MedLine, CAS, SciSearch $\AA$, Current Contents $\AA /$ Clinical Medicine,

Journal Citation Reports/Science Edition, EMBase, Scopus and the Elsevier Bibliographic databases. The manuscript management system is completely online and includes a very quick and fair peer-review system, which is all easy to use. Visit http://www.dovepress.com/ testimonials.php to read real quotes from published authors.

Submit your manuscript here: http://www.dovepress.com/international-journal-of-nanomedicine-journal 